\title{
Optimizing Flight Departure Delay and Route Selection Under En Route Convective Weather
}

\author{
Avijit Mukherjee* \\ University Affiliated Research Center, University of California at Santa Cruz \\ Banavar Sridhar ${ }^{\dagger}$ and Shon Grabbe ${ }^{*}$ \\ NASA Ames Research Center
}

\begin{abstract}
This paper presents a linear Integer Programming model for managing air traffic flow in the United States. The decision variables in the model are departure delays and predeparture reroutes of aircraft whose trajectories are predicted to cross weather-impacted regions of the National Airspace System. The model assigns delays to a set of flights while ensuring their trajectories are free of any conflicts with weather. In a deterministic setting, there is no airborne holding due to unexpected weather incursion in a flight's path. The model is applied to solve a large-scale traffic flow management problem with realistic weather data and flight schedules. Experimental results indicate that allowing rerouting can reduce departure delays by nearly $\mathbf{5 7 \%}$, but it is associated with an increase in total airborne time due to longer routes flown by aircraft. The computation times to solve this problem were significantly lower than those reported in the earlier studies.
\end{abstract}

\section{NOMENCLATURE}

$F \quad=$ set of flights

$J \quad=$ set of sectors

$K=$ set of airports from where flights depart and land

$T \quad=$ number of discrete time intervals, in minutes, that constitute the planning horizon

$R_{f} \quad=$ number of routes available to flight $f \in F$

$N_{f, r} \quad=$ number of NAS resources (sectors and airports) in flight $f$ 's trajectory along route $r$

$P(f, r, i)=i^{t h}$ resource in flight $f$ 's trajectory along route $r$

$l_{f, j}^{r} \quad=$ amount of time flight $f$ is required to spend in sector $j$ along route $r$

$\tau_{f, r} \quad=$ additional flying time of a flight $f$ along route $r$

$d_{f} \quad=$ scheduled departure time of flight $f$

$D_{f}^{r} \quad=$ set of feasible departure times of a flight $f$ along route $r$

$\lambda=$ cost ratio between unit airborne and ground delay of any flight

$S_{j}(t) \quad=$ time-varying capacity of sector $j \in J$

$\operatorname{Dep}_{k}(t) \quad=$ time-varying departure capacity of an airport $k \in K$

$\operatorname{Arr}_{k}(t) \quad=$ time-varying arrival capacity of an airport $k \in K$.

\section{INTRODUCTION}

Convective weather is a major cause of disruption of air traffic flow in the National Airspace System. When convective weather occurs, traffic flow management initiatives, such as the assignment of departure delays and reroutes to aircraft, are implemented. Such initiatives result in delays and cancellation of flights, which imposes

\footnotetext{
* Associate Project Scientist, MS 210-8, Moffett Field, CA 94035, avijit@ucsc.edu, Member of AIAA

† Senior Scientist, MS 210-15, Moffett Field, CA 94035, banavar.sridhar@nasa.gov, Fellow of AIAA

* Research Scientist, MS 210-15, Moffett Field, CA 94035, Shon.R.Grabbe@nasa.gov, Senior Member of AIAA
} 
significant cost on passengers and airlines. Several papers ${ }^{1-9}$ in the literature present optimization models that could enhance the quality, measured in terms of efficiency, equity, safety, and computation time, of air traffic flow management decisions. the objective of the optimization models for the traffic flow management problem is to minimize the total cost of flight delays. The set of constraints in these models include weather-impacted sector capacities, which are significantly lower than the nominal values. The models assign delays and reroutes to a subset of flights such that the capacity constraints of sectors and airports are not violated.

Sridhar et al. (2009) provides a comprehensive review of literature on this topic. ${ }^{10}$ Linear and integer programming ${ }^{11}$ are key techniques applied by researchers to optimize traffic flow management decisions. An advantage of applying linear programming is the availability of state-of-the-art algorithms and software, developed over several decades, to solve large-scale problems. Integer programming models $\mathrm{s}^{1-7,9}$ for the traffic flow management problem have certain limitations. Computation times associated with large-scale problems can be too high for real-time implementation of such models. Recent papers ${ }^{4,9}$ proposed heuristics that can lead to faster computation. Another limitation, cited in Ref. 4, is that delays are assigned to flights without considering if convective weather actually blocks the predicted trajectory of an aircraft. Delays are assigned to flights whose trajectories passes through sectors impacted by weather. Thus, some flights could incur delays although their trajectories are not blocked by weather simply because they fly through the same airspace as those whose trajectories do intersect weather. Such delays could be viewed as unnecessary. A more significant problem reported in Ref. 4 is that the departure delays assigned to some flights may not be enough to prevent their trajectories from intersecting weather. This can happen if the overall delay assignment is such that the reduced sector capacities are not violated, but certain flights reach weather impacted segments of their trajectory due to insufficient amount of departure delays assigned to them. Such situations result in expensive airborne holding and rerouting of flights. Recent studies ${ }^{12}$ have addressed this problem by analyzing the directionality of traffic flow in various sectors, and modifying the sector capacities to better match with weather impact on traffic flows in various directions.

A linear integer-programming model is presented in this paper assigns departure times and reroutes to flights whose trajectories are impacted by convective weather. Departure times are selected from a set of feasible times, provided as inputs to the model, that guarantee weather-free trajectories. Flights whose original flight plans do not pass through weather are flown according to schedule and are not assigned any controlled departure times or reroutes. The sector capacity constraints in the model are the nominal values reduced by the demand created by the uncontrolled traffic. This results in a significantly lower number of binding constraints, which, in turn, tremendously improves the computational time required to solve the integer program. The proposed model does not take into account uncertainty in convective weather, and therefore, is deterministic.

The outline of this paper is as follows. Section II presents an integer-programming model for assigning departure delays and reroutes to aircraft whose trajectories are impacted by convective weather. Experimental setup and data are discussed in Section III. Section IV presents the results. Section V concludes the paper followed by references in Section VI.

\section{AN INTEGER PROGRAMMING MODEL FOR DEPARTURE DELAY AND ROUTE ASSIGNMENT TO FLIGHTS}

In this section, a deterministic optimization model for improving Traffic Flow Management (TFM) decisions, formulated as an Integer Program (IP), is presented. The model assigns departure times and pre-departure reroutes to flights that fly through weather impacted airspace. The departure times are selected from a pre-specified set, provided as input to the model, which ensures flight trajectories remain free of conflict with weather. The set of constraints ensure that airport and sector capacities are not violated. The decision variables, objective function, and constraints of the IP are described below.

\section{A. Decision Variables}

The binary decision variables, $x_{f, t}^{r}$, and a set of auxiliary decision variables, $w_{f, t}^{j, r}$, are defined as follows:

$$
\begin{aligned}
& x_{f, t}^{r}=\left\{\begin{array}{l}
1, \text { if flight } f \text { departs along route } r \text { by time } t \\
0, \text { otherwise }
\end{array}\right. \\
& w_{f, t}^{j, r}=x_{f, t-\sum_{m=1}^{i-1} l_{f, P(f, r, m)}^{r}}^{r}, \text { where } j=P(f, r, i), 1<i \leq N_{f, r} .
\end{aligned}
$$


The decision variables $x_{f, t}^{r}$ are non-decreasing, and constraints are imposed, as discussed later, to ensure that a flight $f$ can fly along only one among $R_{f}$ available routes. Furthermore, the routing decisions are made prior to departure, and hence, a flight stays on its selected route after departing from its origin airport. The auxiliary decision variables, $w_{f, t}^{j, r}$, indicate if $f$ has entered sector $j$ by time $t$ along route $r$. The auxiliary variables are dependent on $x_{f, t}^{r}$. The purpose of defining the auxiliary variables is to make the model presentation and description of objective function and constraints easier.

\section{B. Objective Function}

The objective function minimizes the total cost of departure delay and additional flight time. The additional flight time, which is weighted by the cost ratio $\lambda$, can be incurred due to longer routes flown by some of the flights. The objective function is given by Equation (2) below.

$$
\min \sum_{f \in F} \sum_{r=1}^{R_{f}}\left(\sum_{t \in D_{f}^{r}}\left(t-d_{f}\right)\left(x_{f, t}^{r}-x_{f, t-1}^{r}\right)+\lambda \tau_{f, r} x_{f, \max \left(D_{f}^{r}\right)}^{r}\right)
$$

\section{Constraints}

\section{Non-decreasing decision variables}

Equation (3) imply that if a flight $f$ takes off at time $t$ along route $r$, the decision variables $x_{f, t}^{r}$ assume the value 1 from $t$ onwards.

$$
x_{f, t}^{r}-x_{f, t-1}^{r} \geq 0 \quad \forall f \in F, r \in\left\{1, . ., R_{f}\right\}, t \in D_{f}^{r}
$$

\section{No cancellation constraints.}

The variable $x_{f, \max \left(D_{f}^{r}\right)}^{r}$ is 1 if a flight $f$ departs along route $r$; otherwise, it is 0 . Since the decision variables, $x_{f, t}^{r}$, are binary, Eq. (4) ensures that a flight can depart only along one route among all available ones. Thus, Eq.

(4) ensure that each flight departs along one of its alternative routes, and that no flight is cancelled. Also, since the decision variables are non-decreasing, Eqs. (3) and (4) together imposes the constraints that the chosen route for a flight remains same at all times.

$$
\sum_{r=1}^{R_{f}} x_{f, \max \left(D_{f}^{r}\right)}^{r}=1
$$

\section{Sector capacity constraints}

Equation (5) imposes an upper bound on the number of flights that can occupy the sector during a given timeperiod. The left-hand side of Eq. (5) computes the number of flights occupying sector $j$ at time $t$. The right hand side values $S_{j}(t)$ are provided as input in the optimization model.

$$
\sum_{\left\{(f, r): P(f, r, i)=j, P(f, r, i+1)=j^{\prime}, i<N_{f, r}\right\}}\left(w_{f, t}^{j, r}-w_{f, t}^{j^{\prime}, r}\right) \leq S_{j}(t), \quad \forall j \in J, t \in\{1, . ., T\}
$$

\section{Airport capacity constraints}

Equations (6) and (7) impose departure and arrival rate constraints at airports. Eq. (6) ensures that the number of flights departing an airport $k$ during time period $t$ does not exceed the airport's departure capacity. Similarly, Eq. (7) imposes an upper bound on the number of flights that can land at $k$ during $t$.

$$
\begin{gathered}
\sum_{f: P(f, 1,1)=k} \sum_{r=1}^{R_{f}}\left(x_{f, t}^{r}-x_{f, t-1}^{r}\right) \leq \operatorname{Dep}_{k}(t) \quad \forall k \in K, t \in\{1, . ., T\} \\
\sum_{f: P\left(f, 1, N_{f, 1}\right)=k} \sum_{r=1}^{R_{f}}\left(x_{f, t}^{r}-x_{f, t-1}^{r}\right) \leq \operatorname{Arr}_{k}(t) \quad \forall k \in K, t \in\{1, . ., T\}
\end{gathered}
$$




\section{Decision variables are binary}

$$
\begin{aligned}
& \text { Equations (8) specify that the decision variables } x_{f, t}^{r} \text { and } w_{f, t}^{j, r} \text { are binary. } \\
& x_{f, t}^{r} \in\{0,1\} \text {, and } w_{f, t}^{j, r} \in\{0,1\}
\end{aligned}
$$

\section{DATA DESCRIPTION AND EXPERIMENTAL SETUP}

The deterministic optimization model presented in Section II was applied to solve large-scale instances of TFM decision-making problem. The model was applied to assign departure delays and reroutes to thousands of flights, whose trajectories were impacted due to weather. Realistic traffic and weather data, described in this section, were obtained to perform the experiments.

\section{A. Weather Data}

The Massachusetts Institute of Technology (MIT) Lincoln Lab's Corridor Integrated Weather System ${ }^{13}$ (CIWS) data for June 19, 2007, was used to perform experiments. The convective weather on this day consisted of a squall line that impacted a significant region of the northeastern United States. CIWS data provides 3-dimensional weather information, such as intensity and altitude (i.e., echo tops) of convective weather at various regions of the NAS, at multiple times of day. It also provides $0-2$ hour weather forecasts during those times.

The CIWS data was used to obtain information about weather location and intensity at every 15-minutes from the start of the TFM planning horizon described later in Section III-D. The forecast data was not used, and therefore, the weather information was treated as deterministic. Also, the CIWS Echo-Tops, which provides the altitude of the weather activity, was not used in this study. The CIWS data provides the Vertically Integrated Liquid (VIL) level at 1 square-kilometer regions on a grid. NASA's Future Air Traffic Management Concept Evaluation Tool (FACET) ${ }^{14}$ was used to translate the raw CIWS data into contours, which are basically 2-dimensional polygons, of VIL levels 3 and higher.

Depending on the intensity, size and duration of weather, the number of such polygons could be on the order of a few thousand. Thus, for large-scale problems involving thousands of aircraft and intense weather for long durations, the computation time required for checking if an aircraft's trajectory intersects weather contours could be high. To reduce this computation time, weather contour polygons whose centroids were within a certain (pre-specified) distance were clustered into one subset. A convex hull around the clustered polygons was then generated. As an example, Fig. 1 shows the CIWS weather with VIL level 3 and higher at 6:00 Eastern Standard Time (EST), and the convex hulls drawn across various clusters. The distance threshold used for clustering weather polygons in this case was chosen to be 50 nautical-miles. In the future, experiments could be done to analyze the sensitivity of this parameter. In this example, there were about 900 contour polygons initially present in the weather data, while the number of clusters obtained was 8 . Only two out of eight clusters impacted large regions of the NAS.

Along with reducing computation time, using the convex hulls of weather clusters instead of actual weather contours has another advantage. Although, in the optimization model, weather information is considered deterministic, it is rarely so in reality. In practice, flights are assigned departure delays based on weather forecasts that predict location and intensity of weather constraints several minutes to hours in the future. Due to uncertainty in the forecasts, it may not be practical to assign delays or reroutes to aircraft based on small gaps between weather constraints, and predicted several hours in advance. Using the convex hulls of weather clusters could result in more robust TFM decisions than using actual weather contours.

\section{B. Traffic Data}

The Enhanced Traffic Management System ${ }^{15}$ (ETMS) data for August 24, 2005 was used to generate traffic information required by the optimization model. The weather on this day was relatively mild. This allowed for obtaining information on schedule, trajectories and unimpeded travel times of flights, and traffic loads at various sectors under nominal conditions. The traffic data included take-off times and trajectories (based on track data recorded by radars) of flights that originated from, and/or were destined to, a U.S. airport. The data also included the original and modified flight plans, which consisted of waypoints along a flight's scheduled trajectory, cruise altitude, and ground speed. The ETMS data were read by FACET, and a java-based program using the FACET Application Programming Interface was developed to generate flight-specific information required as input to the optimization model. 


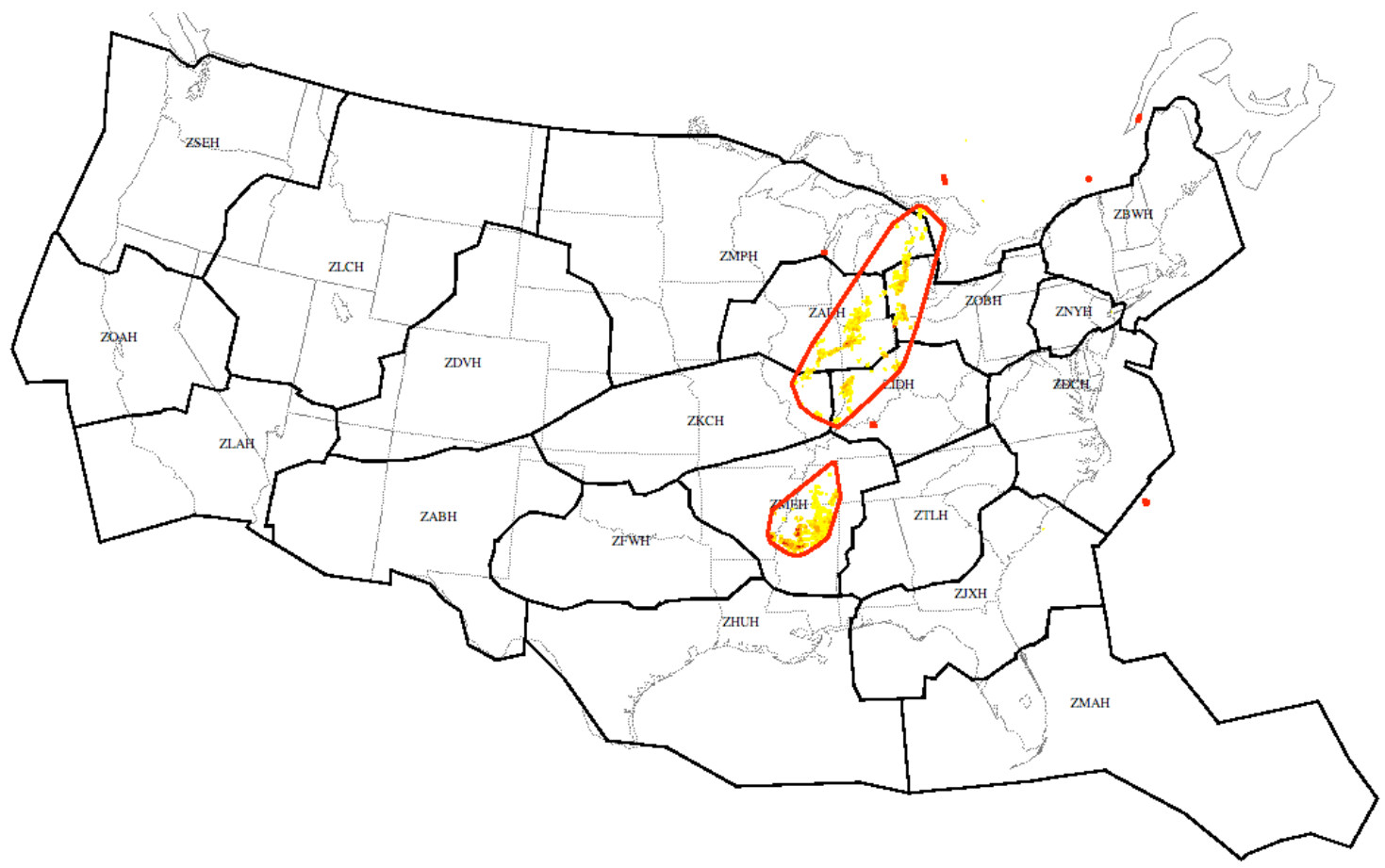

Figure 1: Convective weather and convex hulls of weather clusters at 6AM EST

\section{Alternative Route Generation}

Alternative, weather free, routes for a subset of flights was provided as input to the optimization model, so that pre-departure rerouting could be done. Dijkstra's algorithm was implemented to find the shortest-distance weatherfree route between two airports. Traffic density in the weather-free regions was not considered while generating the alternative routes. However, the sector capacity constraints imposed in the optimization model ensure that sectors are not overloaded. In the classical sense, Dijkstra's algorithm finds shortest path between two nodes in a directed graph when the link costs are static (i.e., not changing over time). An extension of this algorithm, proposed in Ref. 16 , finds the shortest path between two nodes when link costs are dynamic. In the literature, such a network is known to be time-expanded. A required input to the algorithm is the time of departure from the origin node. The shortest path depends on the time of departure and time-varying link costs.

To implement the Dijkstra's algorithm to find the shortest weather free route between two airports, a grid was drawn across the weather impacted region of the NAS. The distance between two adjacent nodes in the grid was set to approximately 20 nautical-miles. The size and location of the grid could be varied to test the sensitivity of the grid geometry on the solutions obtained from the shortest path algorithm. However, this was left as an exercise to be done in the future. The time-varying link costs were generated using the weather convex hulls, described above, and the geometry of the grid. If weather blocked a link during at a particular time-interval, the associated link traversal during that time was set to a large value.

By applying Dijkstra's algorithm, ${ }^{16}$ one alternative, weather-free route was obtained for $70 \%$ of the flights whose primary routes were impacted by weather. Alternative routes were not available for the remaining $30 \%$ of the flights mainly because their departure or arrival airports were impacted by weather for extended periods of time. The alternative route data was provided as input to the TFM optimization model. Figure 2 shows an example of primary (highlighted in blue) and alternative, weather-free (highlighted in green) routes between John F. Kennedy, and San Francisco international airports. 


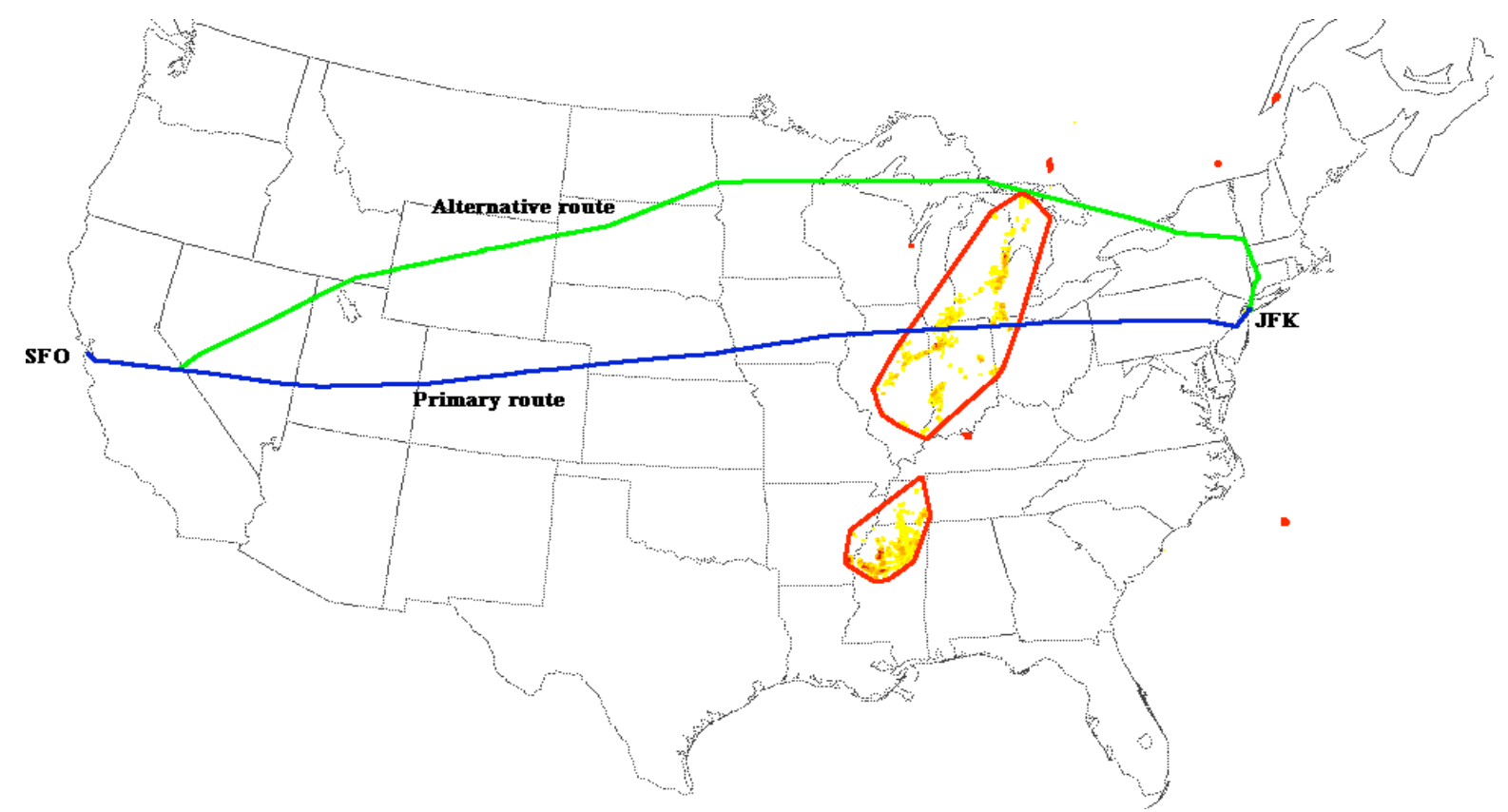

Figure 2: Primary and alternative routes between John F. Kennedy Intl. Airport (JFK) and San Francisco Intl. Airport.

\section{Experimental Setup}

The TFM planning horizon $T$ was chosen to be from 6:00 to 12:00 EST. The planning horizon was divided into 72 discrete time periods, each of 5-minutes duration. Traffic data between 6:00 - 9:00 EST was used to generate the necessary inputs, described below, to the optimization model. Flights that departed or landed during this time interval, or were airborne at the start of the planning horizon were included in the study. In total, there were 8600 flights. As mentioned earlier, CIWS data for June 19, 2007, at every 15-minutes interval starting from 6:00 until 12:00 was used. It was assumed that the weather obtained at the start of an interval remained static during the interval.

FACET was used to generate, for each flight in the system, the set of sectors along the flight's path and the corresponding occupancy times. International flights, flights that were already airborne at the start of the planning horizon, and flights with weather free trajectories were considered as "exempt", which means that they were not assigned any delays or reroutes. Previous studies have applied similar methods to reduce the set of flights and decision variables. ${ }^{17}$ Exempting flights from being assigned delays can cause inequity in the distribution of delay among flights. Such issued are discussed in Ref. 18. From hereon, flights that are not exempt are termed as "controlled" flights. Out of 8,600 flights, about 1,900 were controlled. The set of exempt flights, however, contributed towards sector loads, which ultimately impact the sector capacities that could be allocated to controlled flights. The time-varying demands in a sector that is caused by the exempt flights were subtracted from the sector's Monitor Alert Parameter (MAP) values to generate residual capacities, which were used as constraints (Eq. 5) in the optimization model.

For each controlled flight, a set of feasible departure times was generated as follows. For a potential time of departure of a flight, which started as the flight's scheduled departure time, if the flight could depart on its nominal route without intersecting any of the weather convex hulls, then that time is counted as a feasible departure time. The feasible departure times of a flight depends on its schedule and the location and intensity of weather. The TFM optimization model assigns a flight a controlled departure time within this set. Therefore, it is ensured that trajectories of flights are free of any conflict with weather.

The optimization model was applied to three cases, described below, and the results obtained were compared.

\section{Baseline case}

In this case flights were provided with only one available route and no rerouting decisions were made. The optimization model simply assigned departure delays to flights. Furthermore, in this case no attempt was made to 
ensure that flight trajectories remain free of any conflict with weather. This case is similar to the approach taken in the previous studies such as in Ref. 4. After the model assigned departure delays, a simulation was run in which flights were assigned airborne holding to prevent them from flying through weather. The time-varying capacity of sectors impacted by weather was reduced using a simple weather translation model. The reduced capacity of a sector $j$ at time-period $t$ was derived using Eq. 9. The amount of capacity reduction is proportional to the area of a sector impacted by CIWS weather with VIL level 3 or higher. The available capacity is set to zero if $50 \%$ or more of a sector is covered by weather.

$$
S_{j}(t)=\max \left(0,1-2 \frac{\text { area of CIWS contours in sector } j \text { at time } t}{\text { area of sector } j}\right) M A P_{j}
$$

where,

$M A P_{j}=$ MAP value (i.e., weather-free capacity) of sector $j$.

\section{No-rerouting case}

As in the baseline, each flight was provided with only one available route. The optimization model was applied to obtained flight departure times. The departure times assigned by the model belonged to a pre-specified set, provided as input, which ensuring flight trajectories were free of conflict weather. In this case, flights, after taking off, did not face any airborne holding. The sector capacities used in the constraints were set to the nominal values (i.e., MAP values) reduced by the sector load caused by the exempt flights.

\section{Rerouting case}

In this case, one alternative route was provided to $70 \%$ of the controlled flight as discussed in Section III.C. The optimization model was applied to assign departure delay and pre-departure reroute to flights. The model ensured that the trajectories of flights remained weather-free. As in the no-rerouting case, the sector capacities used in the constraints were set to the nominal values (i.e., MAP values) reduced by the sector load caused by the exempt flights. No airborne holding was necessary in this case. However, additional flight times were incurred due to some aircraft flying on longer alternative routes, which were weather-free.

\section{RESULTS}

This section presents the results when the optimization model was applied to the three experimental cases described above. First, delays are compared between the baseline and no-rerouting cases. These results reflect the benefit, in terms of reduced airborne holding, of ensuring flight trajectories to be free of conflict with weather. Results from the rerouting case exhibits the value of pre-departure routing when severe weather persists for extended periods of time.

\section{A. Delay Comparison between Baseline and No-Rerouting Cases}

When rerouting was not allowed, the average departure delay assigned by the TFM optimization model, presented in Section II, was 29.6 minutes per delayed flight. The total departure delay was approximately 55,000 minutes. The total amount of airborne holding was zero as the departure time of a flight belonged to a feasible set that ensured its trajectory remained weather-free.

In the baseline case, flights receive total delay of 10,000 minutes. While this amount is much less than the norerouting case, significant airborne holding $(108,000$ minutes) is required to prevent flights from flying through convective weather (see Table 1 for delay comparison.) Reducing sector capacities while not ensuring that the 4dimensional trajectories of flights are weather-free results in significant airborne holding, which ultimately offsets the benefits of lower departure delays. Airborne holding is not only expensive due to fuel burnt by aircraft but also is a factor contributing to airspace congestion, controller workload, and can ultimately compromise safety in flight operations.

\begin{tabular}{|l|c|c|}
\hline \multicolumn{2}{|l|}{ Table 1: Delay Comparison } & Baseline Case \\
\hline & 10,000 & No-Rerouting Case \\
\hline Total departure delay (minutes) & 108,000 & 0 \\
\hline Total airborne delay (minutes) & 1000 & 0 \\
\hline $\begin{array}{l}\text { Total departure delay (in minutes) } \\
\text { of flights whose initial flight plan } \\
\text { is weather-free }\end{array}$ & & \\
\hline
\end{tabular}


In the baseline case, approximately 1,000 minutes of departure delay were assigned to those flights whose initial flight plans were free of conflict with weather. In the no-rerouting case, such flights were treated as exempt, and thus received no delays.

\section{B. Results for No-Rerouting Case}

Figure 3 shows the flight tracks of aircraft whose departure delay exceeded 2 hours. The flight tracks are highlighted in red. Also shown in the figure are five Centers - Cleveland (ZOB), Chicago (ZAU), Atlanta (ZTL), Memphis (ZME), and Kansas City (ZKC), which are highlighted in cyan. These five centers incurred significant convective weather activity on the day chosen for this study. As evident from the figure, the primary route of almost all severely delayed flights crossed one of more of these centers. Flights whose primary route did not intersect any weather were exempt, and therefore did not incur any delays.

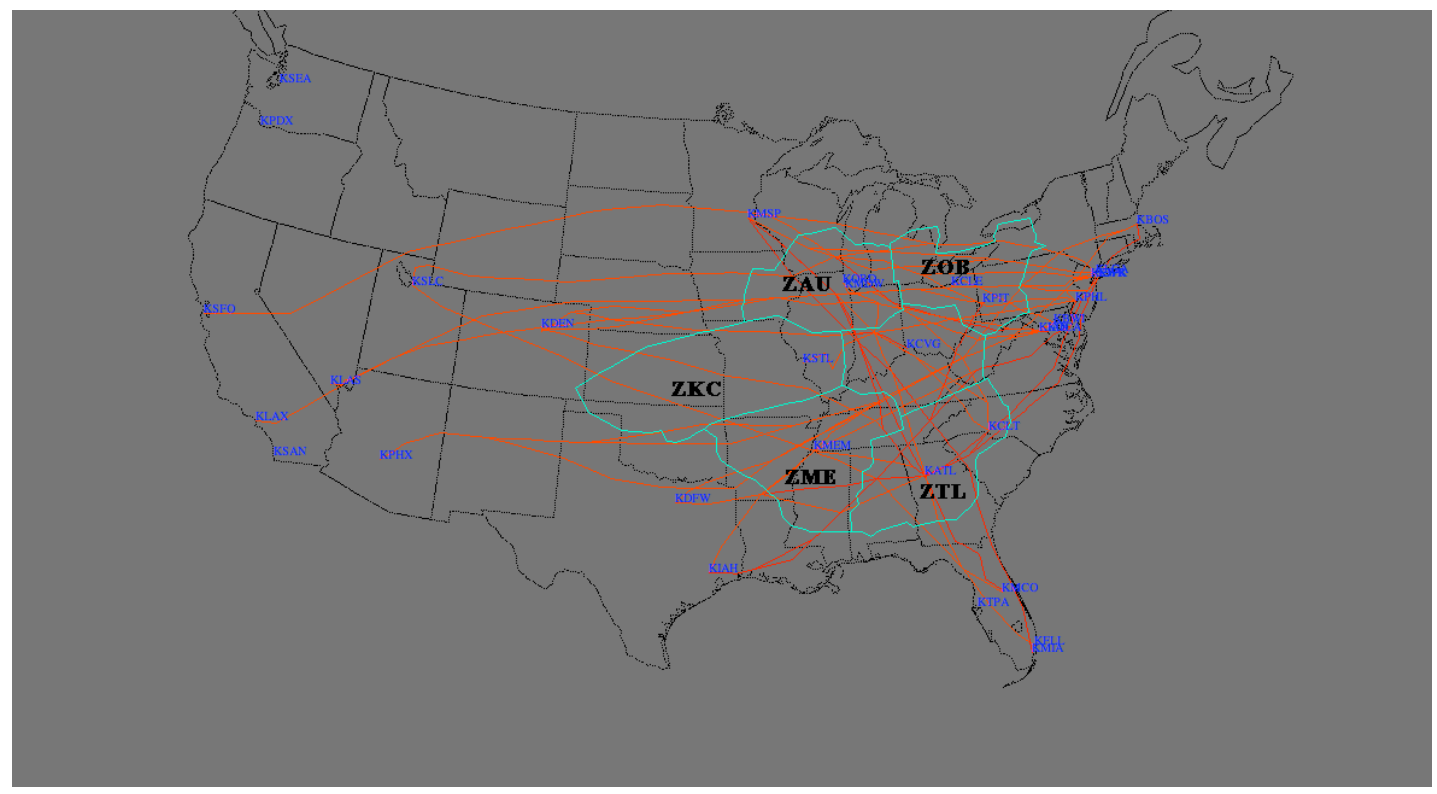

Figure 3: Trajectories of severely delayed flights

The delays assigned to controlled flights by the optimization model results in reduction of sector counts. Thus, the optimization model indirectly reduces the traffic loads in various sectors that are impacted by weather, although the sector capacities provided as input were not reduced by any weather translation model as done in the baseline case. As an example, Fig. 4 shows the time-varying sector loads in the high altitude sector ZOB19 before and after the flight schedules were modified based on optimization. This sectors was significantly impacted by weather. Between 8:30 - 10:00 AM EST, the controlled sector load is lower than the schedule. The delayed flights account for higher sector loads, compared to schedule, during later times.

\section{Results for the Rerouting Case}

When pre-departure rerouting was allowed in the TFM optimization model, departure delays were reduced by $57 \%$ compared to the case where no rerouting was allowed. This reduction in departure delay occurs mainly because about $22 \%$ of flights that faced high departure delays when provided with no alternative route were rerouted with significantly lower delays. The optimization model could assign departure delays to rerouted flights in order to prevent en route sectors from being overloaded. Rerouting is done for flights whose cost of waiting at the origin airport was higher than the additional airborne time due to longer route taken to fly. The cost ratio, $\lambda$, between unit airborne and ground delay was set to 2 . The value of the objective function, which is the weighted sum of airborne and ground delays, reduced by $45 \%$ when rerouting was allowed.

Figure 5 shows the distribution of delays with and without rerouting (i.e., no-rerouting and rerouting cases). When pre-departure rerouting was allowed, about $18 \%$ flights departed on-time compared to the single-route case. Also, rerouting resulted in $4 \%$ drop in the category where flights were delayed between 2-3 hours. In general, with the option to reroute, severity of departure delays reduced. With rerouting, no flights faced departure delayed in 
excess of 3 hours. While the benefit of rerouting is mainly in the reduction of severe departure delays, it also alleviates congestion over weather-impacted airspace by rerouting traffic over weather-free regions of the NAS.
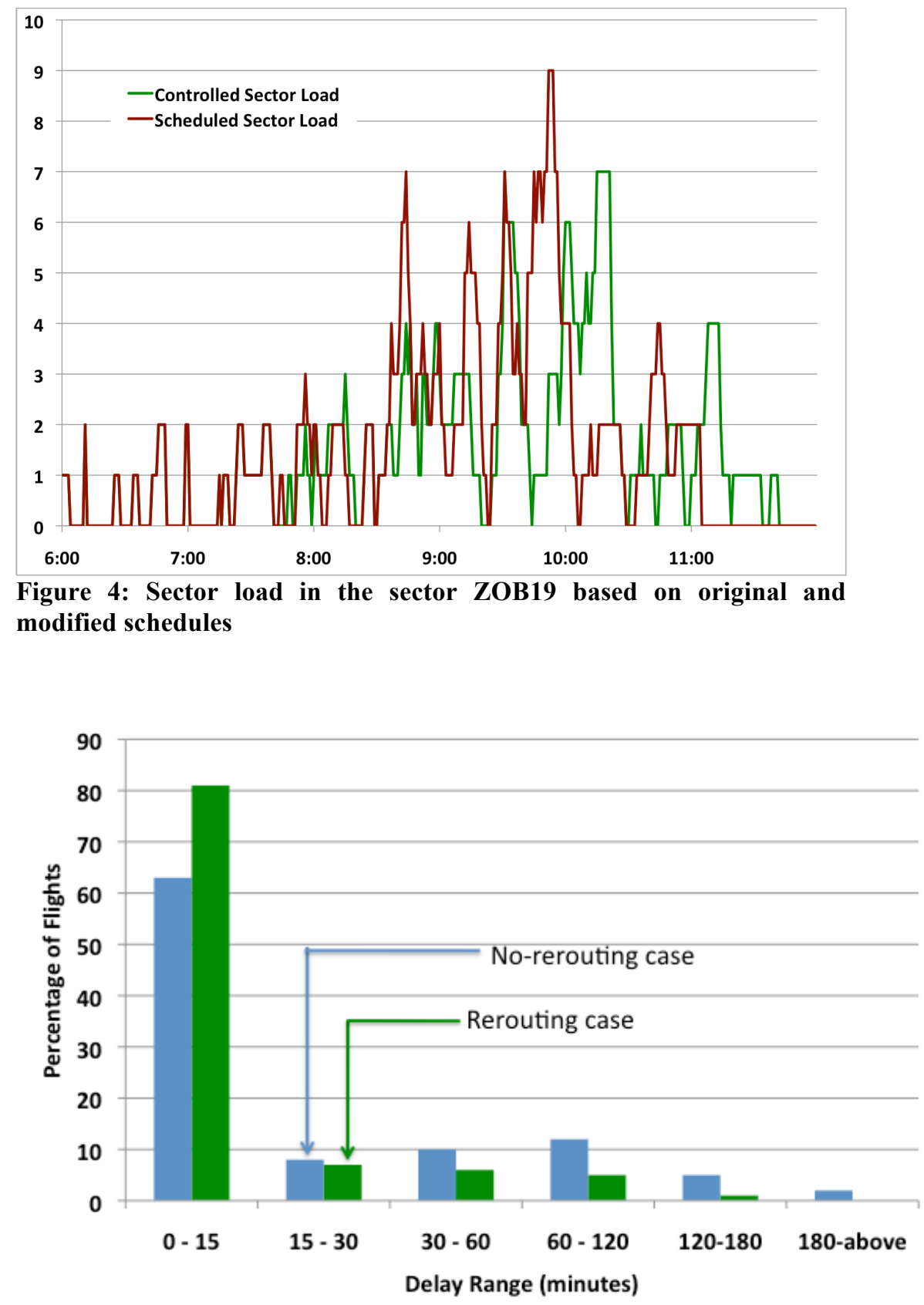

\section{Model Validation}

The average delay of delayed flights on June 19, 2007, reported on the FAA's Operation Systems Network (OPSNET) data was approximately 64.8 minutes per flight. As mentioned before, the average delay for delayed flights from the no-rerouting case was 29.6 minutes per flight. This is about $50 \%$ lower than the observed value. There are two reasons behind this. The optimization model presented here is a deterministic one, where it is assumed that weather occurrence over time is known with certainty. In reality, this is seldom the case. Furthermore, delays in 
the system can occur due to plethora of reasons unrelated to weather. For example, delays due to aircraft mechanical faults are included in the observed delays, whereas it is not in the optimization model. Nevertheless, the comparison between actual and model output delays provide an insight to the potential benefits of applying advanced methods in the traffic flow management decisions.

\section{CONCLUSIONS}

A deterministic optimization model, formulated as a linear Integer Program, for managing air traffic flow was presented in this paper. The decision variables in the model are departure delays and pre-departure reroutes of aircraft whose trajectories are predicted to cross weather-impacted regions of the National Airspace System. The model was applied to solve a large-scale traffic flow management problem with realistic data on weather and flight schedules. Based on the experiments performed in this paper, the computation times to solve the IP for a large-scale problem was significantly low. Future study would focus on computational aspects of the model, modify its formulation, and apply specialized methods to guarantee faster computation.

One of the advantages this proposed model has over existing ones is that it assigns delays to only those aircraft whose trajectories are impacted by weather. However, this may lead to inequitable distribution of delays among flights. The model optimizes the departure delay of flights such that they do not interfere with weather while en route. Therefore, flights do not face airborne holding after taking off. Delay comparison between the baseline and no-rerouting cases shows that significant reduction in airborne holding can be achieved by ensuring that flight trajectories are weather-free. The model allows pre-departure rerouting, which alleviates excessive departure delays. Experimental results indicate that when the cost ratio of airborne and ground delay is 2, there is a $57 \%$ reduction in departure delays, and $45 \%$ in overall delay cost. In general, with the option to reroute, severity of departure delays of flight reduced.

Alternative, weather-free routes between airports were obtained by applying a variant of the Dijkstra's algorithm. In practice, airlines can provide their own choice of primary and alternative routes. Also, the choice of the cost ratio is somewhat arbitrary. Airlines could propose the maximum amount of departure delay of a flight they are willing to take in order to fly through the primary route. The rerouting decision will depend on whether or not a flight can be flown on its primary route with departure delay less than this airline-preferred threshold. The traffic flow management optimization model can easily adopt such airline preferences.

\section{REFERENCES}

\footnotetext{
${ }^{1}$ Bertsimas, D., and Stock Patterson, S., "The Air Traffic Flow Management Problem with En Route Capacities," Operations Research, Vol. 46, pp. 406-422, 1998

${ }^{2}$ Bertsimas, D., and Stock Patterson, S., "The Traffic Flow Management Rerouting Problem in Air Traffic Control: A Dynamic Network Approach,” Transportation Science, Vol. 34, pp. 239-255, 2000.

${ }^{3}$ Grabbe, S., Sridhar, B., and Mukherjee, A., "Central East Pacific Flight Scheduling," Air Traffic Control Quarterly, Vol. 15, pp. 187-210, 2009

${ }^{4}$ Grabbe, S., Sridhar, B., and Mukherjee, A., "Sequential Traffic Flow Optimization with Tactical Flight Control Heuristics," Journal of Guidance, Control, and Dynamics, Vol. 32, pp. 810-820, 2009.

${ }^{5}$ Lindsay, K., Boyd, E., and Burlingame, R., "Traffic Flow Management Modeling with Time Assignment Model," Air Traffic Control Quarterly, Vol. 1, pp. 255-276, 1994.

${ }^{6}$ Mukherjee, A., and Hansen, M., "A Dynamic Rerouting Model for Air Traffic Flow Management," Transportation Research, Part - B, Vol. 43, pp. 159 - 171, 2009.

${ }^{7}$ Mukherjee, A., Grabbe, S., and Sridhar, B., "Arrival Flight Scheduling through Departure Delays and Reroutes," Air Traffic Control Quarterly, Vol. 17, pp. 233 -244, 2009.

${ }^{8}$ Nilim, A., El Ghaoui, L., Duong, V., and Hansen, M., "Trajectory-Based Air Traffic Management under Weather Uncertainty," $4^{\text {th }}$ USA/Europe Air Traffic Management R\&D Seminar, Santa Fe, NM, 2001.

${ }^{9}$ Rios, J., and Ross, K., "Massively Parallel Dantzig-Wolfe Decomposition Applied to Traffic Flow Scheduling," AIAA Journal of Aerospace Computing, Information and Communication, Vol. 7, pp. 32-35, 2010.

${ }^{10}$ Sridhar, B., Grabbe, S., and Mukherjee, A., "Modeling and Optimization in Traffic Flow Management," Proceedings of the IEEE, Vol. 96, pp. 2061-2080, 2009.

${ }^{11}$ Nemhauser, G. L., and Wolsey, L. A., Integer and Combinatorial Optimization, John Wiley and Sons, 1998.

${ }^{12}$ Klein, A., Cook, L., Wood, B., Simenauer, D., “Airspace Capacity Estimation Using Flows and Weather Impacted Traffic Index," IEEE Integrated Communications, Navigation, and Surveillance Conference, 2008.

${ }^{13}$ Evans, J. E., and Ducot, E. R., "Corridor Integrated Weather System," MIT Lincoln Laboratory Journal, Vol. 16, 2006.

${ }^{14}$ Bilimoria, K. D., Sridhar, B., Chatterji, G., and Grabbe, S. R. (2001), "Future ATM Concepts Evaluation Tool," Air Traffic Control Quarterly, Vol. 9, pp. 1-20.
} 
${ }^{15}$ Volpe National Transportation Center (2005), “Enhanced Traffic Management System (ETMS),” Report No. VNTSCDTS56-TMS-002, U.S. Department of Transportation, Cambridge, MA.

${ }^{16}$ Chabini, I., and Lan, S., "Adaptation of the A* Algorithm for the Computation of Fastest Paths in Deterministic DiscreteTime Dynamic Networks,” IEEE Transactions on Intelligent Transportation Systems, Vol. 3, No. 1, pp. 60-74, 2002.

${ }^{17}$ Rios, J., and Ross, K., "Solving High Fidelity Large-Scale Traffic Flow Management Problems in Reduced Time," AIAA International Council of Aeronautical Sciences Conference, Anchorage, Alaska, 2008.

${ }^{18}$ Sherali, H.D., Smith, J.C., and Trani, A.A., “An Airspace Planning Model for Selecting Flight Plans under Workload, Safety, and Equity Considerations," Transportation Science, Vol. 36, pp. 378-397, 2002. 